\title{
Modelling Nonlinear Responses of Resonance Sensors in Pressure Garment Application
}

\author{
Timo Salpavaara and Pekka Kumpulainen \\ Tampere University of Technology, \\ Department of Automation Science and Engineering, \\ Korkeakoulunkatu 3, 33720, \\ Tampere, Finland \\ \{timo.salpavaara, pekka.kumpulainen\}@tut.fi
}

\begin{abstract}
Information on the applied pressure is critical to the pressure garment treatment. The use of the passive resonance sensors would be significant improvement to existing systems. These sensors have nonlinear response and thus require nonlinear regression methods. In this paper we compare three nonlinear modelling methods: Sugeno type fuzzy inference system, support vector regression and multilayer perception networks. According to the results, all the tested methods are adequate for modelling an individual sensor. The used methods also give promising results when they are used to model responses of multiple sensors.
\end{abstract}

Keywords: nonlinear regression, anfis, MLP, support vector regression, pressure sensor.

\section{Introduction}

The pressure garment treatment has potential to improve the healing process of burns and to reduce the swelling. The use of suitable pressure is critical to the treatment and thus, in order to ensure the proper functioning of the pressure garment, this pressure has to be measured. The typical pressure of the issued pressure garment range from 20 to $60 \mathrm{mmHg}$ (millimeters of mercury). The desirable features for the sensors used in this application, besides the obvious good metrological properties, are the small size of the sensor and disposability. The size of the sensor is an issue because under the pressure garment, a thick sensor acts in a similar manner as a pressure bandage and thus increases the pressure locally which leads to a systematic error. The disposable transducers are needed to avoid contamination since the sensors are placed in contact with the skin of the patients. The pressure of the pressure garments can be measured by using an air filled pouch and tubing [1]. The tubing conducts the pressure of the clothing to a separate measurement unit. An alternative method is to use transducers based on the capacitive [2] and piezoresistive [3] principles. However, the electrical wiring is required to connect these transducers to measurement devises. 
A novel approach to measure the pressures under the pressure garments is the inductively read passive resonance sensors [4]. The key advantages of this approach are the wireless connection to the sensor under the garment, the simplicity and the small size of the sensor. The wireless connection makes the system more convenient to use since the electrical wiring or tubing are significant disadvantages especially when the garments are put on. The simple sensor design is an advantage since we aim at a disposable, adhesive bandage-like sensor. In addition, the simple structure of the sensor may enable the use of the new fabrication methods like printing the structures directly on supporting material. Other tested applications of the passive resonance sensors in the field of medicine are intra-ocular pressure sensing [5] and ECGmeasurements [6].

The main idea of this sensing method is to inductively measure the resonance frequency of an LC-resonator with an external reader coil. The measurand affects either the inductance or the capacitance of the sensor and the inductor in the sensor serves as a link coil. One drawback of this method is the nonlinear response of the sensor. The resonance frequency of the LC-circuit is nonlinear by nature. In addition, in many applications the relation between the measurand and the capacitance or the inductance is nonlinear.

Since the responses of the resonance sensors are inherently nonlinear, methods for converting the measured resonance frequencies to the pressure are needed. The lookup tables or calculation of the pressure from electrical and mechanical models are possible but these methods do not really support the idea of disposable sensors. The needed information is impractical to measure, store and deliver. The sensor can be made less nonlinear by stiffening the mechanical structure by the cost of the sensitivity. If the nonlinearity problem can be handled, more possibilities for the structural and fabrication innovations become available. One solution for this problem is to measure the response of the sensor and to identify a nonlinear model. The model can be used to convert the measured frequencies to the measurand. For example neural network model has been used for compensating the nonlinear response of capacitive sensors [7].

In this paper we test three nonlinear regression methods to model the responses of the passive resonance sensors.

\section{Passive Resonance Sensor and Used Read-Out Methods}

The instrumentation in this work consists of a hand-held measurement unit and passive resonance sensors. The measurand in this application is the pressure under the clothing. It is converted to the electrical signal by using pressure dependent capacitors. The pressure on the sensor alters the impedance of the capacitors by reducing the distances between the capacitor plates. This alters the phase response of the resonance sensor. The phase response is measured wirelessly through the inductive link using the hand-held measurement unit. Afterwards, the PC post processing software calculates an estimate for the resonance frequency based on the phase response data. The post processing also includes the compensation algorithm to reduce the error caused by the unknown coupling coefficient of the inductive link. 
The coil and the capacitor of the resonance circuit are created by assembling a dual-layer structure which consists of the PCB (printed circuit board) and PDMS layers (Polydimethylsiloxane). The link coil is etched on PCB. There are two rigid electrodes on the PCB layer which form two pressure dependent capacitors in a combination with the electrode layer on the PDMS. There is also a discrete capacitor on the PCB. The capacitor is needed to tune the sensor to the proper frequency range. The flexible PDMS layer is glued on top of the supporting PCB layer. It has two major features: a cavity which allows the structure to deform and a metal electrode which forms the other half of the pressure dependent capacitors. The electrical equivalent circuit of the tested sensor is similar to earlier studies [4]. The tested sensor and the parts needed for the assembly are shown in Fig. 1.

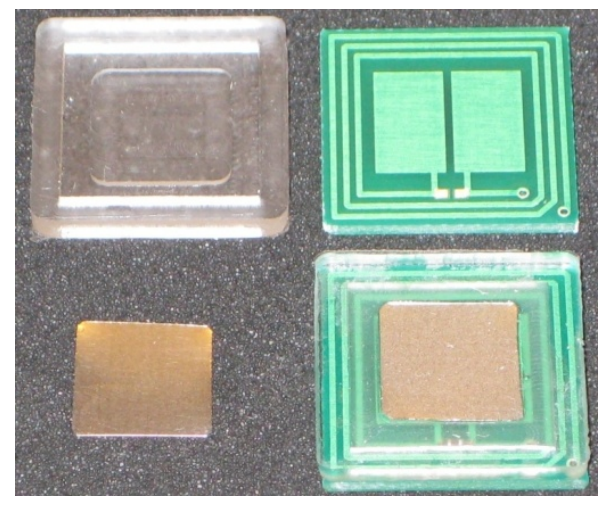

Fig. 1. The used components and the complete pressure sensor

The resonance frequency of the sensor is measured wirelessly through the inductive link with the reader device, which operates like an impedance analyzer [4]. The device sweeps over the specific frequency range and measures the phase response of the resonance sensor. The measurement distance is approximately two centimetres.

The first step of the post processing is to extract the features from the measured phase response data which contains phase values at discrete frequencies. These features are the relative frequency and the relative height of the phase dip or peak depending on the used reader coils and sensors. These features are used to calculate the compensated estimate for the resonance frequency. In the pressure garment application this frequency is compared with the frequency measured without the pressure garment. For the flowchart of the sensing method and for more detailed description see [4].

The described sensing system has a nonlinear response to a pressure stimulus. This is caused by the nonlinearity of the resonance frequency of a LC-resonance circuit

$$
f=1 / 2 \pi \sqrt{L C} .
$$

where $L$ is the inductance and $C$ is the capacitance of the circuit. Furthermore, there is nonlinearity between the capacitance of a plate capacitor and the distance $d$ between the plates. 


$$
C=\epsilon_{0} \epsilon_{r} \frac{A}{d} .
$$

where $\varepsilon_{0}$ is permittivity of vacuum, $\varepsilon_{\mathrm{r}}$ is the relative permittivity of the material and $A$ is the area of the plates. In the tested sensors, the effective capacitance of the resonance circuit is formed as a combination of the constant and variable capacitors. In addition, the relation of the distance between the capacitor plates and the applied pressure is nonlinear, especially, if the deformation of the structure is large. The mutual effect of these nonlinearities depends on the structure of the sensor and the used components and materials. The response of the sensor is also affected by imperfections in parts, materials and assembly.

The nonlinearities and the unknown imperfections of the measurement system make it practically impossible to form an exact physical model for calculation of the pressure from the measured frequency. Therefore we test nonlinear regression to model the response of the sensor based on the calibration measurements. The most important range of the measurand is from 20 to $60 \mathrm{mmHg}$, because the pressure of the proper pressure garments should be within this range. However, at the moment the mechanical properties of the used sensor allow a reliable measurement range from 0 to $45 \mathrm{mmHg}$ only.

In this study we have three sensors, A1, A2 and A3. We have data from two separate measurement cycles from each sensor. Each cycle consists of 11 data points. In addition, we have a series of more dense measurement cycle from sensor A1, which consist of 21 data points. These data are divided into training and test sets in the following case studies.

\section{Nonlinear Regression Methods}

Regression models are used to relate two variables; the dependent variable $y$ and the independent variable $x$ which is typically multivariate. The goal is to find a function $f$ to predict $y$ from values of $x: y=f(x)$. In this application the relationship between the measurand and the sensor response is nonlinear and therefore a nonlinear function $f$ is required. In this paper we compare three nonlinear regression methods that are presented in the following subsections.

\subsection{Sugeno Fuzzy Inference System}

Sugeno, or Takagi-Sugeno-Kang, is a fuzzy inference system that can be used for nonlinear regression [8]. The membership functions of the output are either constant or linear functions. The final output is a weighted average of the output functions, weighted by the membership functions of the inputs. The membership functions can be identified from the data by ANFIS (adaptive-network-based fuzzy inference system), a hybrid learning algorithm, which combines the least-squares method and the backpropagation gradient descent method [9].

In this study we use an ANFIS model with linear output functions. Examples of the system are given in section 4 . 


\subsection{Support Vector Regression}

The basic Support Vector Machine (SVM) is a classifier that uses hypothesis space of linear functions in a high-dimensional kernel-induced feature space [10]. Support Vector Regression, such as, $v$-SVR [11] can be used for modelling nonlinear dependences of continuous data. Parameter $v$ controls the number of support vectors in the model. In this study we use Radial Basis Function (RBF) kernel: $K\left(\mathbf{x}_{i}, \mathbf{x}_{j}\right)=\exp \left(-\not \mid \mathbf{x}_{i}-\mathbf{x}_{j} \|^{2}\right), \gamma>0$. It is versatile and works well in most applications [12].

In order to avoid over fitting, the optimal values for the parameters $v$ and $\gamma$ are selected by cross validation. We use 5-fold cross validation. Parameter values that provide minimum error in the test are selected. In this study we use a software package LIBSVM [13].

\subsection{Multilayer Perceptron Network}

Multilayer perceptron (MLP) networks [14] are very commonly used neural networks. MLP with one hidden layer containing sufficient number of neurons acts as a universal approximator. Therefore they are excellent tools for nonlinear regression. We train the MLP network by using Levenberg-Marquardt method [15].

\section{Case Study 1: Individual Sensor}

The data used in this case study are acquired by measuring the compensated resonance frequencies of the sensor A1 while the pressure on sensors is altered in a test setup. The stimulus is created by funnelling pressurized air through a valve to a container. The pressure in the container is measured with a pressure calibrator (Beamex PC105). This pressure is mediated on the sensor with a rubber film which is attached to the opening of the container. The rubber film simulates the behaviour of the skin in the actual measurement event. The other side of the sensor is fixed to a rigid plate. The resonance frequency values of the data are acquired wirelessly by using the hand-held measurement unit. Each frequency value is an average of more than 100 measured values.

The training data consists of two short measurement cycles from sensor A1. The test data consist of the longer more dense measurement cycle from sensor A1.

\subsection{Nonlinear Models}

We train the ANFIS model with 3 membership functions in the input and output. The membership functions after training are presented in Fig. 2. The lower part depicts the 3 linear output functions. The slope of the functions steepens from function 1 to 3 . The upper part presents the membership functions of the input of Gaussian bell shape. The final output is the average of the linear output functions weighted with the input functions. As the scaled frequency increases towards zero, the weight of the steepest output function also increases producing a steepening curve as seen in Fig. 4. 


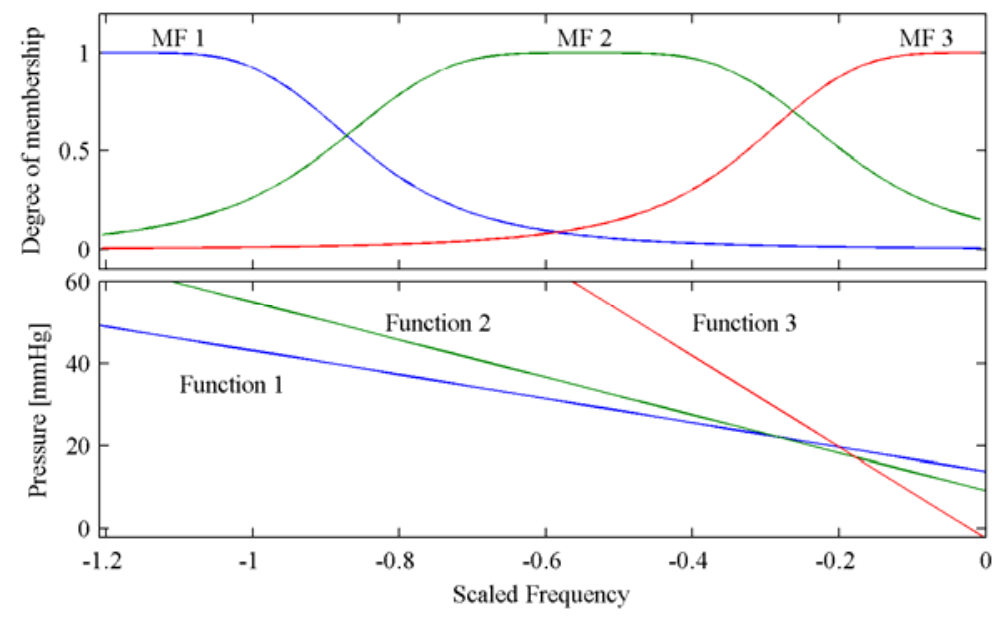

Fig. 2. Membership functions of the anfis model

We use of 5-fold cross validation to find the optimal values for $v$ and $\gamma$ parameters in $v-\mathrm{SVR}$ model. The cross validation error as a function of $v$ and logarithm of $\gamma$ is presented in Fig 3. The minimum is found at values $v=0.8$ and $\gamma=2$. These values are used to train the model using the whole training data set.

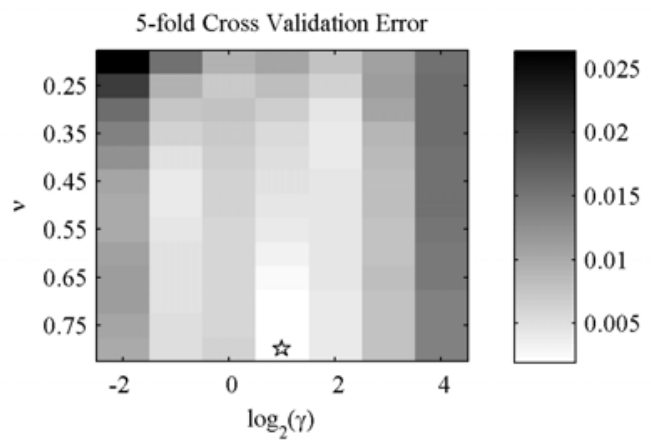

Fig. 3. Cross validation error

We use a small MLP network with one hidden layer with two neurons and sigmoid activation functions. One output neuron has a linear activation function.

\subsection{Results on Training and Test Data}

The trained models are used to estimate the pressure. The results are extrapolated slightly outside the range of the training data set. The results are presented in the left side of Fig. 4. The errors at the measured values in the training data are depicted on the right side. Corresponding results of the test data are presented in Fig. 5. 

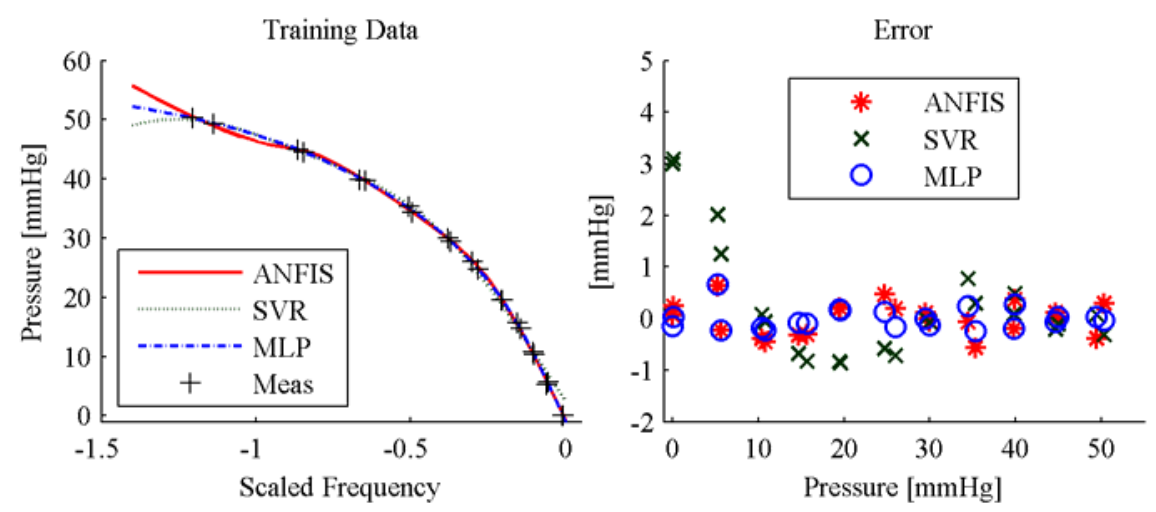

Fig. 4. Estimates and errors for the identification data
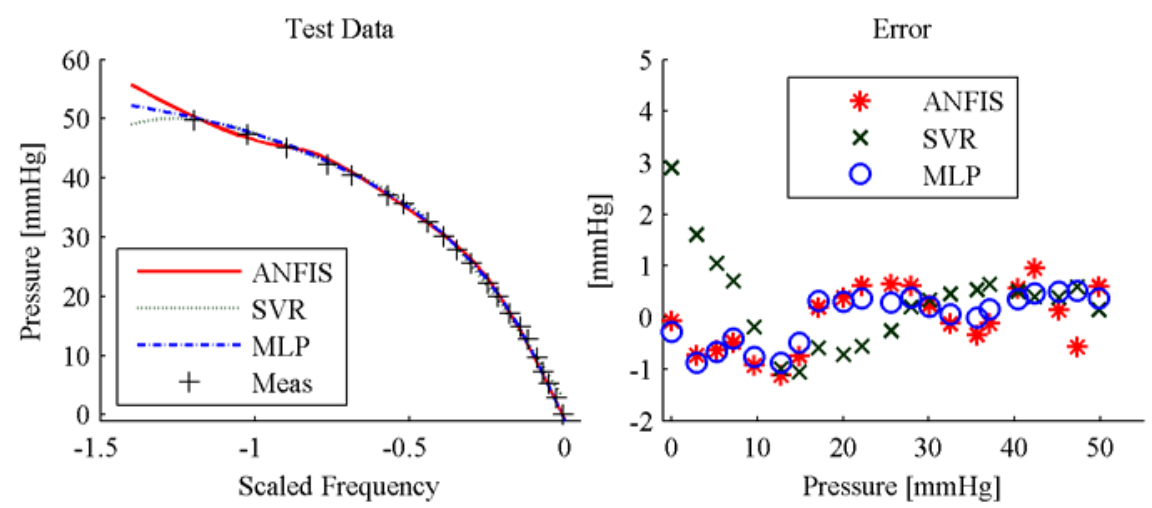

Fig. 5. Estimates and errors for the test data

All the tested methods will provide acceptable results because the repeatability of an individual sensor is good and the identification data and the test data are similar. The error is smaller than $\pm 1 \mathrm{mmHg}$ in most of the test points. Only the model made with SVR method has errors larger than $\pm 1 \mathrm{mmHg}$ at the low pressures. However, this is not significant in this the application. The error of the denser test data has smooth distribution. According to this result, the models can estimate the behaviour of the response between the identification data points. The models start to separate outside the tested range. The use of any of these models outside the tested range is not advisable. The model made with SVR is not monotonic if the shift of the resonance frequency is high. The errors of ANFIS and MLP tend to group and they differ slightly from the errors of SVR.

\section{Case Study 2: Group of Three Sensors}

The data of this case study were measured in a similar manner as in the case study 1 . The identification data in this case consist of two short measurement cycles from 
sensors A1 and A2 The test data are the long measurement cycle from sensor A1 and both short cycles from sensor A3. The data of sensors A2 and A3 contain only short, less dense measurement cycles. According to the results made on case study 1, the repeatability of the sensors is adequate. The short measurement cycle is sufficient to cover the response of the sensor within the tested pressure range. Such a dense coverage of the range as used in the long cycle of A1 is not required for modelling the response.

The measured training data is presented on the left side of Fig. 6. The test data is depicted on the right side of Fig. 6. The estimates of the regression models are included in both.
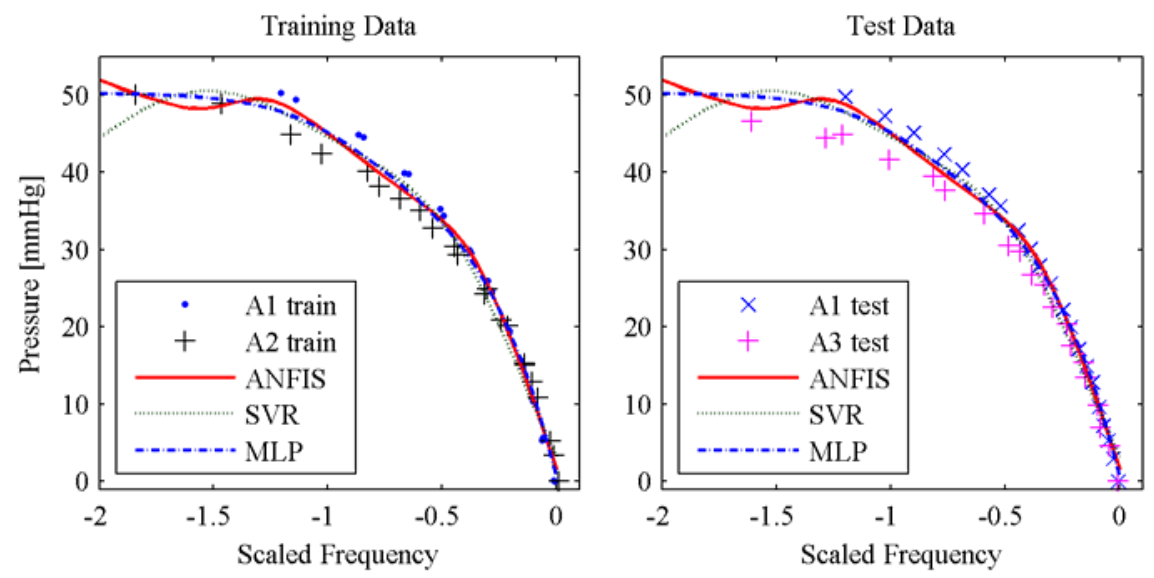

Fig. 6. Training and test data of the case study 2 with the estimates by all 3 regression models
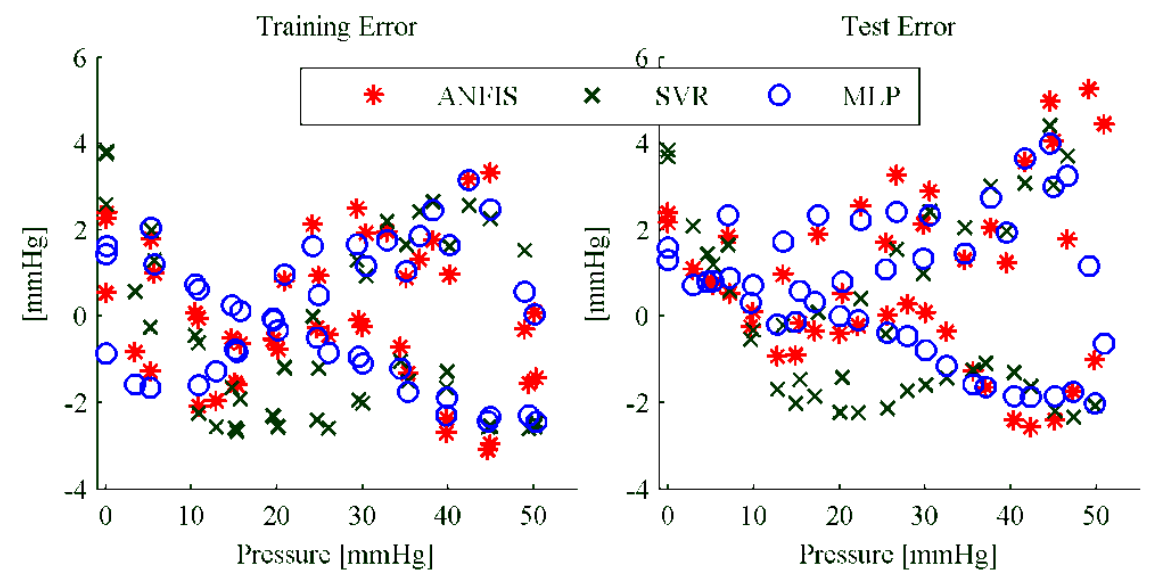

Fig. 7. Errors of the training and test cases 
Sensors A2 and A3 seem to share similar behaviour. Sensor A1 differs from them especially at higher pressure levels. Because the training data contains the same number of measurements from sensors A1 and A2, the estimates of the models are in between them. The models made with ANFIS and SVR are not monotonic if the shift of frequency is high, which limits the usability of these models.

The errors of case study 2 are presented in Fig. 7. The errors are smaller than \pm 2 $\mathrm{mmHg}$ at most of the data points within the pressure range from 5 to $40 \mathrm{mmHg}$. However, especially near the edges of the tested pressure range, the error occasionally rises to over $4 \mathrm{mmHg}$. The errors seem to group according to the tested sensor rather that tested method. The errors are roughly two to three times larger than in case study 1. There is no significant difference between the errors in training and test data. The overall performance of tested regression methods is very similar.

\section{Conclusion}

According to these results, any of the tested methods will make considerable improvement to our earlier attempts to convert the shift of the resonance frequency of the passive resonance sensor to the measurand. The response of the individual sensor can be modelled with accuracy that is more than adequate for the application. The case study 2 shows that having just a single model for converting the shift of resonance frequency to pressure might be sufficient. The errors within the tested range of pressures are smaller than $\pm 2 \mathrm{mmHg}$ in most cases. The errors are considered to occur mainly due the differences in the responses of the tested sensors. The models are useful only at the limited range of the frequency shifts. The frequency shifts outside of this range should not be converted to pressure by using the models. In the future, more efficient and automated fabrication methods will be tested. This will diminish the the differences between sensor responses and it will increase the number of the sensors available for testing. The more abundant data set is needed in order to study if there is a significant difference between the tested regression methods in this application. However, according to these results, the use of the any tested methods will give a realistic tool for the converting the shift of the resonance frequency to a pressure which in turn will encourage the experimenting on the more innovative sensor structures which have nonlinear response.

Acknowledgments. The authors like to thank Mr. Jarmo Verho for the cooperation in the designing of the used readout methods and devices.

\section{References}

1. Van den Kerckhove, E., Fieuws, S., Massagé, P., Hierner, R., Boeckx, W., Deleuze, J.P., Laperre, J., Anthonissen, M.: Reproducibility of repeated measurements with the Kikuhime pressure sensor under pressure garments in burn scar treatment. Burns 33, 572578 (2007)

2. Lai, C.H.Y., Li-Tsang, C.W.P.: Validation of the Pliance X System in measuring interface pressure generated by pressure garment. Burns 35, 845-851 (2009) 
3. Ferguson-Pell, M., Hagisawa, S., Bain, D.: Evaluation of a sensor for low interface pressure applications. Medical Engineering \& Physics 22, 657-663 (2000)

4. Salpavaara, T., Verho, J., Kumpulainen, P., Lekkala, J.: Readout methods for an inductively coupled resonance sensor used in pressure garment application. Sens. Actuators, A. (2011) (in press, corrected proof, March 5, 2011)

5. Chen, P.-J., Saati, S., Varma, R., Humayun, M.S., Tai, Y.-C.: Wireless Intraocular Pressure Sensing Using Microfabricated Minimally Invasive Flexible-Coiled LC Sensor Implant. J. Microelectromech. Syst. 19, 721-734 (2010)

6. Riistama, J., Aittokallio, E., Verho, J., Lekkala, J.: Totally passive wireless biopotential measurement sensor by utilizing inductively coupled resonance circuits. Sens. Actuators, A. $157,313-321$ (2010)

7. Patra, J.C., Kot, A.C., Panda, G.: An intelligent pressure sensor using neural networks. IEEE Trans. Instrum. And Meas. 49, 829-834 (2000)

8. Sugeno, M.: Industrial Applications of Fuzzy Control. Elsevier, New York (1985)

9. Jang, J.-S.R.: ANFIS: Adaptive-Network-based Fuzzy Inference Systems. IEEE Transactions on Systems, Man, and Cybernetics 23(3), 665-685 (1993)

10. Cristianini, N., Shawe-Taylor, J.: An Introduction to Support Vector Machines and Other Kernel-based Learning Methods. Cambridge University Press, Cambridge (2000)

11. Schölkopf, B., Smola, A., Williamson, R.C., Bartlett, P.L.: New support vector algorithms. Neural Computation 12, 1207-1245 (2000)

12. Hsu, C.-W., Chang, C.-C., Lin, C.-J.: A Practical Guide to Support Vector Classification, http://www.csie.ntu.edu.tw/ cjlin/papers/guide/guide.pdf

13. Chang, C.-C., Lin, C.-J.: LIBSVM: a library for support vector machines (2001), http://www.csie.ntu.edu.tw/ cjlin/papers/libsvm.pdf

14. Haykin, S.: Neural Networks. McMillan, New York (1994)

15. NNSYSID Toolbox - for use with MATLAB, http://www.iau.dtu.dk/research/control/nnsysid.html 